\title{
Las influencias de las emociones en el rendimiento académico de los estudiantes de educación primaria
}

\section{The influences of emotions on the academic performance of elementary school students}

DOI: $10.46932 / \mathrm{sfjdv2n3-022}$

Received in: May 1st, 2021

Accepted in: Jun 30th, 2021

\section{Mg. Betty Beatriz Salavarria Barco}

Universidad Cesar Vallejo. Magíster En Diseño y Evaluación de Modelos Educativos. Universidad Tecnológica Empresarial de Guayaquil, Guayaquil-Ecuador

E-mail: bettysalavarria@ hotmail.com

\section{Mg. Nora Mercedes Torres Torres}

Universidad Cesar Vallejo. Magister en Bibliotecología. Universidad Regional Autónoma de los Andes, Ambato-Ecuador

E-mail: n.torrest2309@gmail.com

\section{Mg. Francisco Mera Velázquez}

Universidad Cesar Vallejo. Magister en Educación Superior. Universidad de Guayaquil. Ecuador, franciscomera64@yahoo.com

Magister en Enseñanza de Inglés como idioma Extranjero. Universidad Católica Santiago de GuayaquilEcuador,

E-mail: franciscomera64@yahoo.com

\section{RESUMEN}

La finalidad de este artículo es describir las influencias que tiene el campo emocional en el correcto desempeño de los estudiantes de primaria del establecimiento educativo García Goyena situada en cantón Salitre, provincia del Guayas, Ecuador, variables que han generado mayor interés. En los últimos tiempos, los estudiantes de esta institución educativa han mostrado actitudes hacia las emociones negativas en su comportamiento en clase, otros no prestan atención a las actividades que desarrollan los docentes en clase, entre otras situaciones que llevó a la ejecución de este trabajo de investigación. La exploración se desarrolló con un enfoque cualitativo y no experimental; por el nivel descriptivo bibliográfico. La selección se basó en aspectos como aportes, pertinencia y relevancia. Los efectos de las emociones confirmaron la existencia de deficiencias emocionales en el ámbito de la convivencia por deficiencias en la educación emocional. Por tanto, se presentan tácticas educativas para el desarrollo de la inteligencia emocional, con actividades dirigidas a los docentes responsables del aprendizaje de los alumnos, que son los principales beneficiarios, ya que pueden mejorar sus actitudes en su comportamiento. con sus compañeros, con los profesores y sus familias. Asimismo, se considera que los padres, profesores y la institución educativa en general se beneficiarán de esta propuesta, mejorando la orientación del contenido de aprendizaje.

Se concluyó, proponiendo una visión imperativa de que los estados emocionales inciden en el desempeño académico del estudiante en cualquier nivel educativo y en la educación emocional desde la niñez para el desarrollo de capacidades y habilidades diseñadas para formar estudiantes emocionalmente sanos con el fin de contribuir la excelencia de la enseñanza.

Palabras claves: Aprendizajes, emociones, estudiantes, miedo, rendimiento académico. 


\begin{abstract}
The purpose of this article is to describe the influences that the emotional field has on the correct performance of primary school students from the García Goyena educational establishment located in Salitre canton, Guayas province, Ecuador, variables that have generated greater interest. In recent times, the students of this educational institution have shown attitudes towards negative emotions in their behavior in class, others do not pay attention to the activities carried out by teachers in class, among other subjects. which led to the execution of this research work. The exploration was developed with a qualitative and non-experimental approach; by the bibliographic descriptive level. The selection was based on aspects such as contributions, relevance and relevance. The effects of emotions confirmed the existence of emotional deficiencies in the area of coexistence due to deficiencies in emotional education. Therefore, educational tactics for the development of emotional intelligence are presented, with activities aimed at teachers responsible for student learning, who are the main beneficiaries, since they can improve their attitudes in their behavior. with your classmates, with teachers and their families. Likewise, it is considered that parents, teachers and the educational institution in general will benefit from this proposal, improving the orientation of the learning content.

It concludes by proposing an imperative view that emotional states affect student academic performance at any educational level and emotional education from childhood for the development of abilities, skills and abilities. designed to develop emotionally healthy students in order to contribute to teaching excellence.
\end{abstract}

Keywords: Learning, emotions, students, fear, academic performance.

\title{
1 INTRODUCCIÓN
}

En los tiempos actuales, específicamente en el ámbito formativo, las emociones Desempeñan un papel fundamental en el proceso de enseñanza-aprendizaje, y surgen en los estudiantes dificultades de miedo y ansiedad, que no permiten su total concentración en la realización de tareas, situaciones que los profesionales de la educación deben estar al corriente de forma eficaz. Muchos de los procedimientos a estos problemas no son más que insertar el mundo de las emociones en el currículum, como se muestra (Gutiérrez \& Expposto, 2015). Las emociones tienen una influencia fundamental en el rendimiento académico, actuando directamente sobre el aprendizaje.

Cualquier mediación dentro del espacio pedagógico debe estar guiada por la observancia y la previsibilidad, sin embargo, los estados emocionales que acompañan a este proceso no pueden ser eliminados. Por ello, se formula que para lograr una mayor eficiencia y calidad en el proceso de enseñanza-aprendizaje, el conjunto de todas las extensiones que intervienen en este proceso se considera de suma importancia en el ámbito emocional,, En este sentido, (Linares, 2017) afirma que: el proceso de aprendizaje es un asunto de interacción y por lo tanto son necesarias ciertas capacidades y habilidades que permitan que se desarrolle en los escenarios más favorables, en los que gestionamos las emociones. . adecuadamente. 
Según Salmurri (2014) las exploraciones de las emociones están en el origen de un cambio de prototipo y de conocimiento sobre cómo pueden manifestarse en los estudiantes, por lo que es importante saber cómo afrontarlas; algunos antecedentes que se ocupan de ellos. La teoría de Gardner de la inteligencia múltiple, la neurociencia y su contribución a la flexibilidad del cerebro y la relación tanto en pensamiento como en emoción; teoría de la complejidad y la publicación de Goleman de 1995, Emotional Intelligence. Cómo hablar sobre las emociones y el desempeño escolar incluye temas como el manejo emocional y la autorregulación. Cuando se trata de emociones, aunque son muchas, el sexo se ha definido como bases automáticas e inconscientes que pueden ser ampliamente distinguidas: alegría, enfado, rabia, miedo, sorpresa y tristeza, porque existen en cada uno de los individuos y refiere este autor que son identificables.

Con el objetivo de conocer qué emoción pueden tener las emociones en el ámbito de la educación, se realiza este estudio, cuyos resultados se recogen en tanto Pulido \& Herrera (2015), donde se encuentran las relaciones entre emociones como el miedo y capacidad emocional. En este apartado se hace énfasis en el impacto de las variables emocionales en el rendimiento académico, la variable más representativa en el ámbito educativo.

Al mencionar la concepción de emociones, teorías que se enfocan en tres componentes importantes, se toman como punto de partida: el cognitivo experimental, el componente fisiológico y la respuesta conductual adaptativa, este trabajo se centra en las emociones de miedo, las cuales son vistas como emociones con contenido claramente negativo o desagradable.

Es importante tener en cuenta las consecuencias adversas que pueden clasificarse en este tipo de estados emocionales negativos relacionados con la negatividad de muchas situaciones o su exposición, especialmente al sufrir graves molestias durante la infancia. Por tanto, este período puede considerarse un nivel de riesgo. Así lo confirman Gómez \& Ortiz, Casas \& Ortega Ruiz (2016). "Entre los entes que influye claramente en la prevalencia de este tipo de problema emocional es la edad".

La flexibilidad del cerebro de los estudiantes es un constituyente fundamental para considerar el adecuado desarrollo de la gnosis y la afectividad para lograr una personalidad completa e imparcial (intelectual, física, emocional y social). El procedimiento más natural y eficaz es el "juego de interacción", que se utiliza para expresar y gestionar las emociones, incorporando nuevos recursos que se pueden generalizar a la vida diaria. Aprender adecuadamente de todo esto los beneficiará en todos los espacios de su vida. De manera similar, la definición de Goleman de inteligencia emocional interpersonal, que se compone de empatía y habilidades sociales, es comparable a las nociones actuales de habilidades sociales que toman en cuenta el papel crucial de la empatía (Roca, 2005). la comprensión emocional les proporciona confianza y credibilidad en sus prácticas diarias; Construirán y mantendrán relaciones 
agraciadas, sabrán comunicar en todo momento lo que requieren, lo que piensan y opinan, y sabrán tener en cuenta las impresiones de los demás. Así se predispondrán para estar informado, asumir desafíos y aprender; Tendrán alta autoestima, podrán favorecer su estado de anímico, y dispondrán de recursos para la resolución de conflictos.

En particular, las emociones se categorizan en mediadoras de las famosas funciones ejecutivas (inhibición, planificación, toma de decisiones y flexibilidad) y atención, que influyen efectivamente en la capacidad de meditación, manteniendo la atención y creatividad, etc., en definitiva: en todo lo relacionado a favorecer el rendimiento académico.

\section{MATERIAL Y MÉTODOS}

Este artículo se enmarcó en el arte de exploración, se centraliza en el campo de la educación primaria con el objetivo de describir y analizar las influencias que tienen las emociones en el beneficio académico de los estudiantes de instrucción primaria tomando como referente todo lo relacionado con el nivel de logro significativo con este grupo de alumnos, así como la correlación existente entre las variables, fue aplicado en la Institución Educativa Fiscal García Goyena en el Cantón Salitre de la Provincia Guayas-Ecuador. Año 2019.

La metodología se basó en la recopilación bibliográfica sobre el tema de las emociones y es la población de estudio de alumnos de nivel elemental e intermedio, docentes y representantes legales de esta institución educativa. El estudio se realizó mediante el método bibliográfico descriptivo, se realizó una búsqueda en las plataformas digitales de fuentes bibliográficas bajo el criterio de rigurosidad científica en el área de Ciencias educativas. La variable estudiada corresponde al Estado emocional en el rendimiento académico. Como tecnología más importante para la compilación de información, se utilizó la indagación avanzada a través de descriptores clave, relacionados con emociones, educación, rendimiento académico.

De esta forma, los criterios que se mantuvieron para la elección del material consultado incluyeron la relación con el tema de investigación, año de publicación desde 2013 hasta la actualidad.

\section{RESULTADOS}

La educación emocional tiene como objetivo dar mejoras las habilidades emocionales de los estudiantes, es decir, conocer, regular e integrar sus emociones como herramienta que influye en el aprendizaje. En este contexto, cabe decir que El informe Delors citado por (UNESCO, 1996) sugiere que la educación en el siglo XXI debe desempeñarse en torno a cuatro ejes primordiales, denominados las cuatro columnas de la educación: 1) Aprender a aprender, los beneficios aprovechar las oportunidades de 
educación para la educación permanente; 2) Aprenda a permitirle a la persona lidiar con muchas y diferentes situaciones. 3) Aprender a ser, a actuar con autonomía, juicio y responsabilidad personal, y 4) Aprender a convivir, trabajar en proyectos conjuntos y gestionar conflictos. Con respecto a los ejes básicos de la educación y la contextualización de esta investigación, en el marco del rol del Estado como responsable de la educación de la infancia y la juventud, la política estatal de política social en Ecuador aceptó la investigación universal sobre la educación como un derecho humano y en Constitución de la República, (2008), la educación se establece como un derecho de todos los individuos y una obligación necesaria del Estado. Los artículos 26 y 28 del texto constitucional, respectivamente, establecieron la educación como un área prioritaria de las políticas públicas. posteriormente, el Plan Nacional del Buen Vivir (2017-2021) se propuso "construir un régimen educativo con acceso masivo de excelente calidad". Además de expresarlo buscamos la felicidad como un bien superior, se lo entiende como el objeto deseable.

En cuanto al análisis de la indagación recopilada, se evidencia que las mismas propuestas teóricas convergen sobre el estado emocional y su correlación con el rendimiento académico de los estudiantes. De estos saberes perpetrados se podría finiquitar del análisis y reflexiones que los estados emocionales negativos perturban el rendimiento académico. Miñaca, Hervás \& Laprida (2013), afirman “que las emociones se manifiestan como funciones biológicas del sistema nervioso, consignadas a generar conductas que aseguren la armonía y supervivencia al medio o contexto", se podría exteriorizar, que las emociones son particularidades inherentes en el ser humano, por lo que, el niño o la niña desde pequeños no debe instruirse, sino saber identificarlas y comprenderlas con eficacia ya que del manejo de las emociones depende el éxito o fracaso en el aprendizaje. Debiéndose ser énfasis Para las emociones, sería beneficioso saber distinguir lo positivo de lo negativo, ya que las emociones positivas corresponden a sensaciones agradables o contextos en los que se pueden encontrar emociones como alegría, satisfacción o sorpresa. Por el contrario, las emociones negativas se asocian con sensaciones o situaciones desagradables, incluidas emociones como la tristeza, la ira o el miedo. Así lo afirma (Rojas, 2019), "las emociones son vitales en el aprendizaje. Por lo tanto, el conocer cómo se manejan y qué beneficios tienen en los procesos educativos son fundamentales." De este modo, en el contexto educativo es necesario tomar en cuenta la influencia de las emociones en el buen rendimiento académico para así dar cumplimiento a los objetivos educacionales.

De igual forma Linares (2017) se dice que el proceso de aprendizaje es una interacción implícita y por lo tanto requiere de ciertas destrezas y habilidades que le permitan desarrollarse en los escenarios más favorables donde aplicamos adecuadamente el manejo de las emociones. 
Otro estudio de (Marcué \& González, 2017) sugiere que: El rendimiento académico y el nivel de emociones que experimentan los escolares antes de sus exámenes son factores que influyen en el nivel de entusiasmo en los estudiantes que se manifiesta como se refleja en declaraciones cognitivas, fisiológicas y motoras. El resultado de esta investigación promueve la conciencia de los efectos del estado emocional en los estudiantes.

Actualmente, según (González, 2015) diversos estudios afines en el campo de la educación han encontrado que las emociones son la clave del proceso de enseñanza-aprendizaje y una pieza fundamental para quienes enseñan (docentes) y quienes aprenden (estudiantes). Por tanto, debemos considerar la emoción y el conocimiento como una pareja indisoluble. Según estas afirmaciones, las emociones afectan el aprendizaje y, por tanto, están directamente relacionadas con el rendimiento académico del alumno.

Las fuentes bibliográficas estudiadas dan los resultados de que la investigación consultada coincide en encontrar una correlación entre el estado emocional y el rendimiento académico. Así, los estados emocionales favorables conducen a puntuaciones altas y, por el contrario, los estados emocionales desfavorables influyen en la consecución de puntuaciones bajas, coincidiendo con el hecho de que la inteligencia emocional debe desarrollarse en el entrenamiento para reafirmar y reorientar la visión , asignaciones y valores personales y familiares., profesionales y ciudadanos, a través de preguntas activas, simulaciones y visualizaciones de situaciones que afectan la vida, los sueños y los principios en los que se basan las acciones de cada persona, con estrategias educativas para el desarrollo de la inteligencia emocional, interacción cuando la inteligencia familiar aumenta, aumenta el rendimiento académico de los estudiantes, mejorando de este modo sus puntuaciones, destacando los esfuerzos que la comunidad educativa está realizando para lograr aprendizajes significativos, y debe estar orientada hacia la formación humanística. calidad y calidez.

Todas las deducciones anteriores son controvertidas con los resultados de este estudio, ya que encontraron una agrupación positiva entre la ocurrencia de emoción y el rendimiento académico.

\section{DISCUSIÓN}

En cuanto al análisis de la información recolectada, se puede observar que la misma proposición teórica converge sobre el estado emocional y su relación con el rendimiento de los estudiantes. De estas encuestas se puede deducir de los análisis y reflexiones que los estados emocionales influyen mucho en el desempeño escolar. Esta afirmación coincide con las derivaciones presentadas por (Pulido, 2018) quien señaló que existen relaciones entre los estados emocionales y el rendimiento académico. Así, cuando el rendimiento académico se bloquea en los niveles más altos de ansiedad o miedo, se otorgan las calificaciones más bajas. Por otro lado, las calificaciones más altas se dan en el estado de bienestar. Por 
ello, la investigadora indica que se deben tomar en cuenta y fortalecer las capacidades emocionales para optimar el asunto de aprendizaje en el sistema educativo.

Otro estudio que muestra cómo el estado emocional influye en el desempeño de los estudiantes es el presentado por (Marcué \& González, 2017) donde estos autores señalan que la ansiedad o el miedo son factores que afectan el desempeño académico de los estudiantes. En todos los niveles, estudio principalmente a nivel de licenciatura. Los resultados de esta investigación promueven la conciencia del impacto que tienen los estados emocionales de los estudiantes en su crecimiento y desarrollo como profesionales.

Es importante resaltar que la necesidad de la educación emocional se tomó en cuenta desde temprana edad para que el alumno adquiera habilidades emocionales que le permitan controlar las emociones, las cuales, siendo inherentes a la naturaleza del ser humanos, sin embargo, son inevitables, se pueden controlar. Es así como (Alvis, 2016) argumenta que es un hecho que se ha comenzado a priorizar un reconocimiento total de lo importante que es la adquisición de habilidades emocionales por parte de las personas, lo cual es fundamental para el desarrollo humano de todos.

Asimismo, (Ortíz, 2017) presentó un estudio realizado en la Universidad de las Islas Baleares, sobre la importancia de la educación emocional en el desarrollo integral de los estudiantes de primaria. A partir de sus hallazgos, afirma que: La educación emocional es una parte principal de la educación de cualquier persona y especialmente de niños. Se requiere prácticas continuas para volverse emocionalmente competente. En consecuencia, este prototipo de aprendizaje debe empezar en casa, estar motivado desde los primeros años de existencia y estar presente en todo momento.

\section{CONCLUSIONES}

La realización de esta investigación permitió revelar las influencias que tienen los estados emocionales sobre el desempeño académico de los estudiantes. En tal virtud, diversos estudios han justificado que las emociones de los estudiantes son mecanismos categóricos en la base de su aprendizaje: cuando las emociones son favorables y el estado de bienestar está presente principalmente en el contexto del estudiante, los resultados académicos son generalmente satisfactorios. Por el contrario, las emociones desfavorables conducen a tasas de rendimiento bajas.

La escasez de educación emocional en las instituciones educativas tiene malas consecuencias para los estudiantes y docentes. Como resultado de esta investigación, la inteligencia emocional surge como una respuesta en el área de la educación para satisfacer una serie de implicancias que se manifiestan en la sociedad presente, como son los inconvenientes de conducta y violencia. 
Es necesario rodear en la educación elementos que contribuyan en las aptitudes emocionales, como son el desarrollo de creatividad, el optimismo, la constancia y el autocontrol, entre otras. Tradicionalmente, la docencia se ha ocupado de aspectos epistemológicos en los niños, pero es inevitable desplegar, desde este contexto, los componentes de la Inteligencia Emocional para que se conviertan en seres humanos con capacidades para enfrentarse a la existente sociedad competitiva, materialista y poco afectiva.

De esta forma, las emociones son la base de las acciones de aprendizaje y por tanto la jerarquía de educación emocional en el ambiente educativo debe verse como una parte esencial de la educación de las personas y especialmente de los niños y niñas y adolescentes. para el desarrollo de aptitudes, habilidades y competencias dirigidas a producir estudiantes emocionalmente sanos para mejorar la eficacia de la educación. 


\section{REFERENCIAS BIBLIOGRÁFICAS}

Alvis, J. (2016). Habilidades Emocionales como Factor Fundamental del Desarrollo Infantil Integral. Una Mirada Crítica al Marco de Política Pública Educativa. . Universidad Distrital Francisco José de Caldas. Bogotá. Colombia

Constitución de la República (2008). Sistema Legislativo del Ecuador. Registro Oficial 449 del 20 de octubre

Duro, E. (2017). Desarrollo Emocional. Clave para la Primera Infancia, una Aproximación al Desarrollo Emocional Temprano. Madrid, España: Fondo de las Naciones Unidas por la Infancia. UNICEF.

Estrada, A. (2018). Estilos de aprendizaje y rendimiento académico. Revista Boletín

Redipe, 7(7), 218-228. Recuperado de: https://revista.redipe.org/index.php/1/article/view/536

Goleman, D. Inteligencia emocional. Editorial Kairos. 2002.

González, Z. (2015). La Emoción, motor para el aprendizaje. Uruguay: Comunidad de Educadores para la Cultura Científica. Iberoamérica divulga. IBERCIENCIA.

Gómez-Ortiz, O., Romera, E.M. y Ortega-Ruiz, R. (2016). Parenting styles and bullying. The mediating role of parental psychological aggression and physical punishment. Child Abuse and Neglect, 51, 132143.

González, Z.(2015). Emotion, an engine for learning. Uruguay: Community of Educators for Scientific Culture. Iberoamerica discloses. IBERCIENCIA.

Gutiérrez, M., \& Expósito, J. (2015). Autoconcepto, dificultades interpersonales, habilidades sociales y conductas asertivas en adolescentes. Revista Española de Orientación y Psicopedagogía, 26 (2), 42-58. doi: 10.5944/reop.vol.26.num. 2.2015.15215

Lastre, K., López, L. \& Alcázar, C. (2018). Relación entre apoyo familiar y el rendimiento académico en estudiantes colombianos de educación primaria. Psicogente, 21(39), 102-115. Recuperado de:

http://doi.org/10.17081/psico.21.39.2825

Ley orgánica de educación intercultural del Ecuador. (2018) Disponible en:

https://www.evaluacion.gob.ec/wp-content/uploads/downloads/2015/06/Anexo-b.-LOEI

Linares. (2017). Educación Emocional y Convivencia...¿¿ YAhora qué? Red de Centros de Educación Responsable. Región de Murcia. España

Miñaca L, M. I., Hervás T, M., \& Laprida M, I. (2013). Análisis de programas relacionados con la educación emocional desde el modelo propuesto por Salovey\& Mayer.

Marcué, P., \& González, R. (2017). Las Emociones y el Rendimiento Académico: La Ansiedad Ante los

Exámenes en Estudiantes Universitarios. Monterrey. México: Instituto Tecnológico de Estudios Superiores de Monterrey.

Ortíz, R. (2017). La Importancia de la Educación Emocional en el Desarrollo Integral del Alumno de Primaria. Islas Baleares: Universitat de les Illes Balears. 
Pulido Acosta, F., \& Herrera Clavero, F. (2017). La influencia de las emociones sobre el rendimiento académico. Ciencias Psicológicas, 11(1), 29 - 39. doi: 10.22235/cp. v11i2.1344. recuperado de: file://C:/Users/betty/AppData/Local/Temp/Emociones_en_educacion_como_las_emociones_cognicio1.pdf

Pulido, F., \& Herrera, F. (2017). La Influencia de las Emociones sobre el Rendimiento Académico. Ciencias Psicológicas. Vol.11. Nro.1. Universidad de Granada, España

Plan Nacional del Buen Vivir 2017-2021. (s.f.). Secretaria Nacional de Planificación y Desarrollo. Senplades. Obtenido de http://www.planificacion.gob.ec

Rojas, M. (2019). Importancia de las Emociones en el Aprendizaje. Valencia: Universidad Internacional de Valencia, España.

Salmurri, F., Royo, M., Carpena, A., Sala, J., Marzo, L. y Albaladejo, M. (2014). Aprender y educar con bienestar y empatía. (P. Darder, Ed., M. L. Urritia, Trad.). Recuperado de: http://0site.ebrary.com.millenium.itesm.mx/lib/consorcioitesmsp/detail.action?docID=10914571

UNESCO (1996). La Educación Encierra un Tesoro. Madrid, España: Santillana. Ediciones UNESCO. 